\title{
Everyone gets involved: fostering a high quality teaching and learning culture in the NHS
}

\author{
Philip McElnay, Danya Bakhbakhi, Jane Sansom
}

University Hospitals Bristol

\begin{abstract}
The aim was to create an environment where doctors at all levels felt like an important part of the education team.

Two teaching fellows were appointed to deliver teaching, encourage participation, and quality-assure teaching. An innovative electronic logbook named Teaching Log (T-Log) was developed to reliably "record and reward" teaching occurring at all career levels. A mentor scheme for medical students was established. Mentor training was provided for junior doctors. A near-peer teaching programme was developed for final year medical students on important skills required to be a safe junior doctor.
\end{abstract}

Between September 2013 and January 2014, 83 doctors at our institution logged 657 teaching episodes using the electronic T-Log. 23\% were F1s, $14 \%$ were F2s, $24 \%$ CT1-2s. $36 \%$ of sessions were delivered to 3 rd year medical students and $36 \%$ were delivered to 5 th year students. $24 \%$ were small group seminars, $26 \%$ were bedside teaching sessions, $12 \%$ were lectures, $5 \%$ were simulation sessions and $9 \%$ were clinical skills. $20 \%$ of sessions were delivered to a single student. $100 \%$ of respondents (15) agreed that T-Log was easy to use. $100 \%$ agreed that TLog was useful. $100 \%$ agreed that they would continue to use T-Log.

A survey found the mean score for usefulness of the mentor scheme to be 9.1 (1=not useful, $10=$ very useful). Students saw their mentors on average 6.5 times during a 10 week period. Confidence scores for all key areas of the curriculum were increased following implementation of the scheme. $100 \%$ of students would recommend this scheme to next year's final year students and $100 \%$ of mentor respondents ( $n=8$ ) would participate in the scheme again.

Rewarding those who contribute to teaching provides incentive to further increase the quality of education provided to students. T-Log rewards teaching activity. It also provides useful data at an individual level or at institutional level.

\section{Problem}

Medical students often describe coming to a large city centre teaching hospital as a daunting experience. It can feel anonymous. With approximately 200 medical students attached to our hospital in each academic year it can be a challenge to ensure each individual student feels part of the team. This can be further compounded by the move away from the "firm" system in many UK medical schools.

We aimed to foster a high quality teaching environment where everyone feels included, junior doctors feel rewarded for their teaching efforts and ultimately where everyone feels involved in medical education. In our city centre, tertiary referral teaching hospital, we wanted to change the experience of education from a daunting one to a rewarding one.

\section{Background}

Providing patients with safe, high quality care requires in-depth knowledge, exceptional skill, and professional attitudes from medical students and doctors. The role of senior doctors in education is vital. However the experiences, skills and attitudes that junior doctors can share with students are recognized as invaluable in educating the next generation of doctors.(1)

We aimed to increase the quantity and quality of education that medical students can gain from junior doctors. We did this with our project: "Everyone gets involved: fostering a high quality teaching and learning culture in the NHS."

\section{Baseline measurement}

The qualitative feedback from students who had previously been attached to the hospital was analysed. It highlighted the fact that while students found the quality of individual teaching sessions to be good, they often felt like they "had no single person to report to" or "had no one to mentor them" or "were anonymous in such a large hospital."

\section{Design}

The project's main tools were:

1. Funding was sought for two full-time clinical teaching fellows (CTFs) at the Trust. CTFs were appointed to deliver teaching, encourage participation from juniors, and quality 
assure and evaluate junior teaching. Feedback from students was sought about how the trust could make their experience more rewarding. Appointing CTFs was regarded by a large proportion of students as a logical way of making their education more fulfilling

2. T-Log.org.uk: an innovative, user-friendly electronic teaching log book was developed by the CTFs to reliably "record and reward" teaching occurring at all career levels. We discussed with junior doctors about how we could encourage participation and reward those who participate. Doctors often reported that they were turning up at interview with large quantities of paper to prove they had participated in teaching. These were regarded as difficult to accumulate and that if the education team could develop an "easier way" to do this then it would be seen as benefit to doctors. Doctors accessed T-Log using smartphones, tablets, or computers. They are rewarded with a quarterly PDF report of their teaching and a bronze, silver, or gold award for their commitment to education. It was also conceived to evaluate the success of the scheme at an institutional level

3. We introduced a near-peer mentor scheme for all 3rd and 5th year students. Each student was grouped with 2-3 F2s or core trainees. Having discussed with other junior doctors, this was decided to be the best way to ensure that a student always had access to a mentor. For instance, if one doctor was on leave or on nights then they still had someone in the hospital who was a point of contact

4. Mentor training was provided to junior doctors. This outlined the medical school curriculum and what the mentor scheme involved. It also highlighted how to get help if either they or their student had any concerns. They were asked to deliver at least two teaching sessions for medical students as part of their mentor role

5. A near peer evening teaching programme for final year students on relevant skills and attitudes required of a junior doctor was delivered by F1-F2s, primarily on the speciality in which they were currently working.

The project was implemented over a period of 12 months, and will be continued for the foreseeable future.

The key to the sustainability of this project is a culture of reward and involvement that sees everyone feeling like an important part of the educational environment of the hospital. The ongoing input from clinical teaching fellows will be key to the project's successful delivery in the future. The education team has secured ongoing funding for these key members of staff.

\section{Strategy}

PDSA cycle 1:

We initially reviewed the qualitative feedback from students from previous attachments. We realised they felt they needed a mentor figure within the hospital. We hoped to ask doctors to act as mentors for these students but were unsure as to which level of doctor would be best for the role. We surveyed students to ask them what level of doctor they would prefer to have as a mentor.
The results demonstrated that they would prefer an $\mathrm{SHO}$ level doctor, with $69 \%$ of students responding stating they would prefer a doctor at F2-CT2 level.

PDSA cycle 2:

We arranged a meeting with core trainees and F2 doctors and asked whether they would like to participate in a mentor scheme. We thought that the majority would be keen to participate, and indeed they were, with 42 doctors volunteering for the role. However, feedback demonstrated that they would like to be rewarded with evidence for their portfolio. We proposed the idea of an electronic teaching log for doctors to log their teaching activity. This would result in a PDF certificate being generated at the end of the year for the doctor. It would prevent doctors turning up to interviews with reams of paper to prove they teach. It would provide everything in one, user-friendly format that can be printed out when required. This was warmly received.

PDSA cycle 3:

We predicted that many doctors didn't have formal teaching qualifications. We wanted to ensure the teaching that was provided was of high quality and relevant therefore we introduced a mentor training programme. This was well attended by mentors, with all mentors receiving training.

PDSA cycle 4:

We designed an electronic teaching log (T-Log). We sought feedback from the doctors about how they felt about the log. 100\% of respondents (15) agreed that T-Log was easy to use. 100\% agreed that T-Log was useful. 100\% agreed that they would continue to use T-Log. Qualitative feedback from doctors and other members of the educational team demonstrated that special recognition for those who go "above and beyond" in their role as teacher/mentor may be a good way to foster an environment where participation is rewarded. We introduced bronze, silver, and gold awards for teaching based on these markers.

\section{Results}

We measured the interventions' effects by collating feedback on each of the projects tools, from students and from teachers. We also analysed the user data entered into the electronic teaching log.

There were 19 final year medical student responses to an evaluation of the mentor scheme. The survey found the mean score for usefulness of the mentor scheme to be 9.1 (1=not useful, $10=$ very useful). Students saw their mentors most frequently $1 \mathrm{x} /$ week and on average 6.5 times during a 10 week period. Confidence scores for all key areas of the curriculum were increased following implementation of the scheme. $100 \%$ of students would recommend this scheme to next year's final year students and $100 \%$ of mentor respondents $(n=8)$ would participate in the scheme again.

T-Log data showed that there were 762 teaching episodes logged 


\section{BMJ Quality Improvement Reports}

by 112 users of the teaching log between September 2013 and March 2014. Ninety-nine doctors were using the T-Log to record their teaching activity and 13 final year medical students who were participating in near peer educational activities. $75 \%$ of all the $\mathrm{F} 1$ doctors in the trust were using T-Log, $51 \%$ of F2 doctors and $38 \%$ of core trainees.

Of all of the sessions logged (figure 1) 173 were logged by F1s, 101 by F2s, and 176 by core trainees. By the middle of the year consultants and senior trainees had also begun to use the tool.

Of the 762 teaching episodes, the majority were delivered to the 3rd and 5th year medical students, who were assigned mentors in the initial instances. 262 were delivered to 3 rd years and 252 to 5 th years. The tool proved so useful that it was also adopted by those teaching in 1st, 2nd and 4th year courses, and in postgraduate sessions such as journal clubs. It was also found that students were receiving teaching in small groups, with over two thirds of teaching occurring in groups of 10 or less. This enables teaching to be more tailored to the students needs.

See supplementary file: ds3327.jpg - "Figure 1- Grade of doctor logging each individual session in T-Log"

\section{Lessons and limitations}

This project highlighted the fact that junior doctors are highly able and willing teachers. The knowledge, skills, and attitudes they can impart to medical students can be invaluable.

It is important to maintain the balance between juniors and seniors delivering teaching. Each have important roles to play and must feel rewarded for their contribution.

We have encountered small problems with the electronic teaching log. It is costly to implement a "login" feature which allows doctors to print their own log. However, the flip-side of this cost is that time involved in the administration of T-Log certificates is reduced.

In the long term, this project relies on the ongoing high profile of education within the trust. Key to this will be ongoing funding for clinical teaching fellows, which has been secured by the academy.

\section{Conclusion}

Rewarding those who contribute outstandingly to teaching provides incentive to further increase the quality of education provided to students. T-Log is an innovative approach enabling doctors to keep reliable records of teaching experience and rewarding those who go "above and beyond." It can provide useful personal data for ARCP and revalidation as well as enabling improved evaluation of teaching at an institutional level.

\section{References}

1. Rodrigues J, Anshuman S, Mitchell A et al. The South-east Scotland Foundation Doctor Teaching Programme - Is "near-peer" teaching feasible, efficacious and sustainable on a regional scale? Med Teach. 2009;(31):e51-e57

\section{Declaration of interests}

Nothing to declare.

\section{Acknowledgements}

Suzan Fowweather, Sue Bamford, Philip Collings, Michelle Raeburn. 